\title{
Convergence analysis of the fixed-point method with the hybrid analytical modeling for 2-D nonlinear magnetostatic problems
}

\author{
Citation for published version (APA): \\ Ceylan, D., Friedrich, L. A. J., Boynov, K. O., \& Lomonova, E. A. (2021). Convergence analysis of the fixed-point \\ method with the hybrid analytical modeling for 2-D nonlinear magnetostatic problems. IEEE Transactions on \\ Magnetics, 57(2), [9201167]. https://doi.org/10.1109/TMAG.2020.3024539
}

DOI:

10.1109/TMAG.2020.3024539

Document status and date:

Published: 01/02/2021

\section{Document Version:}

Accepted manuscript including changes made at the peer-review stage

\section{Please check the document version of this publication:}

- A submitted manuscript is the version of the article upon submission and before peer-review. There can be important differences between the submitted version and the official published version of record. People interested in the research are advised to contact the author for the final version of the publication, or visit the DOI to the publisher's website.

- The final author version and the galley proof are versions of the publication after peer review.

- The final published version features the final layout of the paper including the volume, issue and page numbers.

Link to publication

\footnotetext{
General rights

- You may freely distribute the URL identifying the publication in the public portal. follow below link for the End User Agreement:

www.tue.nl/taverne

\section{Take down policy}

If you believe that this document breaches copyright please contact us at:

openaccess@tue.nl

providing details and we will investigate your claim.
}

Copyright and moral rights for the publications made accessible in the public portal are retained by the authors and/or other copyright owners and it is a condition of accessing publications that users recognise and abide by the legal requirements associated with these rights.

- Users may download and print one copy of any publication from the public portal for the purpose of private study or research.

- You may not further distribute the material or use it for any profit-making activity or commercial gain

If the publication is distributed under the terms of Article $25 \mathrm{fa}$ of the Dutch Copyright Act, indicated by the "Taverne" license above, please 


\title{
Convergence Analysis of the Fixed-Point Method with the Hybrid Analytical Modeling for 2-D Nonlinear Magnetostatic Problems
}

\author{
D. Ceylan, L. A. J. Friedrich, K. O. Boynov, and E. A. Lomonova \\ Department of Electrical Engineering, Eindhoven University of Technology, 5612 AZ Eindhoven, The Netherlands
}

\begin{abstract}
This paper presents the convergence analysis of the fixed-point method (FPM) to model the nonlinear magnetic characteristics of a 2-D magnetostatic problem. In this study, FPM is used as the iterative nonlinear solver of the hybrid analytical modeling (HAM) technique for the accurate computation of the magnetic field distribution. The benchmark consists of a stator with excitation windings, an airgap, and a slotless mover. The relative errors between two successive iterations are calculated using different error estimators: the attraction force on the mover, the Fourier coefficients defined in the airgap, the magnetic flux density, and the magnetic scalar potential distributions. The effect of the number of mesh elements and harmonics on the accuracy and computational cost of the model is investigated for different levels of magnetic saturation. It is observed that the maximum rate of change in the relative difference of attraction force during the iterations is found to be 0.52 under the magnetic saturation. In addition, the absolute error of the attraction force between the developed hybrid model with FPM and the finite element method (FEM) is achieved to be 0.18\%, while HAM has approximately three times less number of degrees-of-freedom compared to FEM.
\end{abstract}

Index Terms-Hybrid analytical modeling, fixed-point method, nonlinear magnetic characteristics, convergence analysis.

\section{INTRODUCTION}

$\mathbf{T}$ HE fast and accurate computation of magnetostatic problems with nonlinear magnetic characteristics requires the coupling of the electromagnetic modeling with a nonlinear solver. The most popular approach to these problems is using the combination of the finite element method (FEM) and the Newton-Raphson method (NRM). There exist many commercial software packages with the capability of taking the nonlinear magnetic characteristic into account using these methods [1], [2]. However, since the derivation of the Jacobian matrix in NRM is challenging, the fixed-point method (FPM) can be considered as an alternative to model the nonlinear magnetic properties. FPM is a very strong candidate especially for the magnetic hysteresis and eddy current problems because of its robust algorithm and stable convergence [3], [4]. Since FPM does not use the Jacobian matrix of the nonlinear equations, it does not suffer from the instability caused by the derivative [5]. In addition, FEM suffers from its large computational cost due to a large number of mesh elements in the airgap. As an alternative to FEM, a relatively new and less time-consuming modeling method called hybrid analytical modeling (HAM) is proposed in [6]. HAM uses the magnetic equivalent circuit (MEC) theory in the stator and rotor, while the airgap is modeled using Fourier analysis (FA). MEC and FA are coupled by interface conditions at the interface. The main advantage of HAM compared to the classical FEM is its low computational cost because of its mesh-free airgap.

In [7], HAM is applied to a flux switching permanent magnet machine with a nonlinear solver developed using a golden section search (GSS) algorithm, where the implementation of neither HAM nor GSS are explained in details. The modeling formulation of HAM is discussed in detail in [8] and [9], assuming that the soft-magnetic materials have constant permeability. A nonlinear hybrid analytical model is developed in [10] to investigate the cogging force of linear permanent magnet machines. However, the consideration of

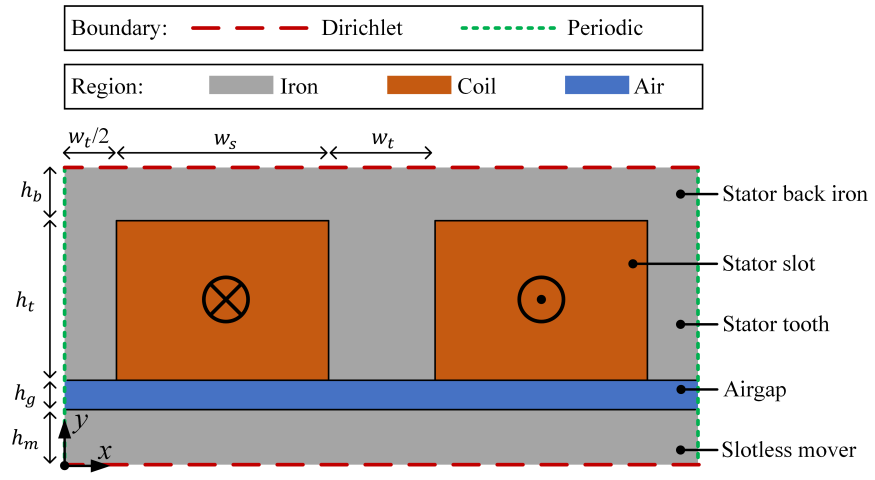

Fig. 1. Analyzed E-core benchmark.

magnetic saturation is not explained in detail. Moreover, the recent improvements on HAM are discussed in [11] with a focus on increasing the accuracy and computational speed. Lastly, Bao et al. present a nonlinear HAM with FPM including the general modeling formulation in [12]. However, the convergence analysis of FPM used in HAM has never been discussed in the literature.

In this paper, an E-core benchmark is modeled using HAM coupled with FPM in a 2-D Cartesian coordinate system. The convergence of FPM is investigated regarding the aforementioned error estimators considering the number of harmonics, mesh refinements, and saturation levels.

\section{BENCHMARK}

The analyzed benchmark is shown in Fig. 1 where $h_{b}, h_{t}, h_{g}, h_{m}, w_{t}$ and $w_{s}$ are 1, 3, 0.5, 1, 2 and $4 \mathrm{~mm}$, respectively. This benchmark is a simplified version of a reluctance machine structure. The soft-magnetic material of the iron regions is selected as $\mu$-metal. While Dirichlet boundary conditions are imposed on the top and bottom, periodic boundary conditions are applied to the left and right edges of the geometry. 


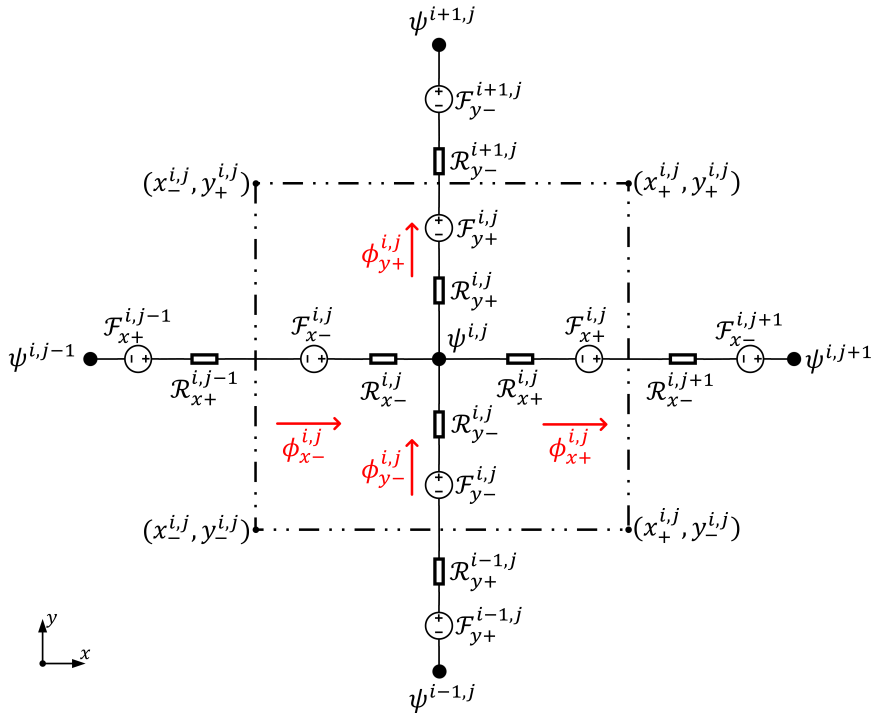

Fig. 2. Single mesh element in a MEC region.

\section{Modeling Method}

In this section, the electromagnetic modeling of the benchmark using HAM and the development of a nonlinear solver using FPM for HAM are discussed.

\section{A. Hybrid Analytical Modeling (HAM)}

HAM uses the strong coupling between the magnetic equivalent circuit (MEC) theory and Fourier analysis (FA). While the mover and stator are modeled using MEC, FA is used to calculate the magnetic field distribution in the airgap.

\section{1) MEC Region}

MEC regions are divided into mesh elements to create a reluctance network as implemented in [13]. The schematics of a single mesh element that is not located on a special boundary is given in Fig. 2 with its neighbor nodes. In Fig. $2, \psi, \phi, \mathcal{R}$, and $\mathcal{F}$ represent the magnetic scalar potential, the magnetic flux, the magnetic reluctance, and the magneto-motive force (MMF), respectively. In HAM, the flux conservation equation is used for each mesh element

$$
\phi_{x-}^{i, j}+\phi_{y-}^{i, j}-\phi_{x+}^{i, j}-\phi_{y+}^{i, j}=0,
$$

where $\phi_{x-}^{i, j}$ is expressed by

$$
\phi_{x-}^{i, j}=\frac{\psi^{i, j-1}-\psi^{i, j}+\mathcal{F}_{x+}^{i, j-1}+\mathcal{F}_{x-}^{i, j}}{\mathcal{R}_{x+}^{i, j-1}+\mathcal{R}_{x-}^{i, j}} .
$$

The other three flux terms, $\phi_{x+}^{i, j}, \phi_{y-}^{i, j}$, and $\phi_{y+}^{i, j}$, of (1) are expressed in the same way. $\mathcal{R}$ values in (2) are calculated using the dimensions of the mesh element, its relative permeability $\left(\mu_{r}\right)$, the permeability of free space $\left(\mu_{0}\right)$, and the stack length of the benchmark $(l)$

$$
\mathcal{R}_{x+}^{i, j}=\mathcal{R}_{x-}^{i, j}=\frac{\left(x_{+}^{i, j}-x_{-}^{i, j}\right)}{2 \mu_{r}^{i, j} \mu_{0}\left(y_{+}^{i, j}-y_{-}^{i, j}\right) l} .
$$

In [14], it is explained that current related MMF distribution in Cartesian coordinate system can be defined either in $x$ direction $\left(\mathcal{F}_{y \pm}^{i, j}=0\right)$ or $y$-direction $\left(\mathcal{F}_{x \pm}^{i, j}=0\right)$. In this study, $x$ direction is selected as the MMF distribution direction. While
$\mathcal{F}_{x \pm}^{i, j}$ is imposed to be zero on the interface between stator slots and airgap, it is linearly increased starting from zero in the stator slots in $y$-direction until the boundary between stator slot and stator back iron. In the stator back iron $\mathcal{F}_{x \pm}^{i, j}$ is constant and equal to the value on the boundary between slot and back iron, which is defined as

$$
\mathcal{F}_{x+}^{i, j}=\mathcal{F}_{x-}^{i, j}=\frac{\left(x_{+}^{i, j}-x_{-}^{i, j}\right) J_{s} h_{s}}{2} .
$$

In addition, for the mesh elements located on the Dirichlet boundary condition, (1) is modified. While $\phi_{x+}^{i, j}$ is forced to zero for the ones on the upper Dirichlet boundary, $\phi_{x-}^{i, j}$ is zero for the ones on the lower Dirichlet boundary. Moreover, for the ones located on the periodic boundary condition, (1) is still valid. To apply the periodic boundary condition, the last element, $N$, of each row, $(i, N)$, is connected to the first element of the row, $(i, 1)$.

\section{2) FA Region}

Unlike the stator and rotor, the airgap is modeled using Fourier analysis (FA). In [15], the magnetic flux density is expressed in $x$ - and $y$-direction as follows

$$
\begin{array}{r}
B_{x}^{\mathrm{FA}}(x, y)=\sum_{n=1}^{N_{h}}\left[\left(a_{n} e^{w_{n} y}+b_{n} e^{-w_{n} y}\right) \sin \left(w_{n} x\right)+\right. \\
\left.\left(-c_{n} e^{w_{n} y}-d_{n} e^{-w_{n} y}\right) \cos \left(w_{n} x\right)\right], \\
B_{y}^{\mathrm{FA}}(x, y)=\sum_{n=1}^{N_{h}}\left[\left(c_{n} e^{w_{n} y}-d_{n} e^{-w_{n} y}\right) \sin \left(w_{n} x\right)+\right. \\
\left.\left(a_{n} e^{w_{n} y}-b_{n} e^{-w_{n} y}\right) \cos \left(w_{n} x\right)\right] .
\end{array}
$$

In (5) and (6), $N_{h}$ is the number of harmonics while $a_{n}, b_{n}, c_{n}$, and $d_{n}$ are the Fourier coefficients. Moreover, $w_{n}$ is the spatial frequency which is a function of the width of the periodical section, $\tau_{p}$, and harmonic index, $n$

$$
w_{n}=\frac{2 \pi n}{\tau_{p}} .
$$

\section{3) Interface between MEC and FA Regions}

The equations for the coupling $\mathrm{MEC}$ and FA regions are derived in [9]. A brief summary of the equations used in this study for the interface is presented in this section. The coupling between MEC and FA models is obtained by two boundary conditions

- Continuity of the magnetic flux density normal to the interface,

- Continuity of the magnetic scalar potential at the interface.

In order to equalize the magnetic flux densities normal to the stator-airgap interface, the flux conservation equation, (1), is modified to

$$
\phi_{x-}^{i, j}+\phi_{y}^{\mathrm{FA}}(j)-\phi_{x+}^{i, j}-\phi_{y+}^{i, j}=0,
$$

where $\phi_{y}^{\mathrm{FA}}(j)$ is the total magnetic flux entering the related mesh element from the airgap. It is expressed as

$$
\phi_{y}^{\mathrm{FA}}(j)=l \int_{x_{-}^{(i, j)}}^{x_{+}^{(i, j)}} B_{y}^{\mathrm{FA}}(x, y) d x
$$


By combining (8), (9) and (6), the boundary condition for the continuity of the magnetic flux density normal to the statorairgap interface is obtained as

$$
\begin{aligned}
& \phi_{x-}^{i, j}-\phi_{x+}^{i, j}-\phi_{y+}^{i, j}=-\frac{l}{w_{n}} \sum_{n=1}^{N_{h}}\{ \\
& {\left[\left[\sin \left(w_{n} x_{+}^{i, j}\right)-\sin \left(w_{n} x_{-}^{i, j}\right)\right]\left[-c_{n} e^{w_{n} y_{b}}-d_{n} e^{-w_{n} y_{b}}\right]\right]-} \\
& {\left[\left[\cos \left(w_{n} x_{+}^{i, j}-\cos \left(w_{n} x_{-}^{i, j}\right)\right]\left[a_{n} e^{w_{n} y_{b}}+b_{n} e^{-w_{n} y_{b}}\right]\right]\right\},}
\end{aligned}
$$

where $y_{b}$ is the $y$-axis position of the stator-airgap interface with respect to the coordinate system defined in the FA region. The same boundary condition is applied to the airgap-rotor interface. Moreover, since the MMF distribution on the statorairgap interface is zero,

$$
\vec{H}=-\nabla \psi
$$

is valid on the interface to implement the continuity of the magnetic scalar potential. Considering the continuity of $\psi$ on the stator-airgap interface and (11), another boundary condition is introduced:

$$
H_{x}^{i, j}=H_{x}^{\mathrm{FA}}\left(x, y_{b}\right) .
$$

In order to have the required number of equations or degreesof-freedom (dof $=N_{x} N_{y}+4 N_{h}$ ), (12) is converted into spatial frequency domain with the same harmonic orders as in the FA region as proposed in [9]. In dof, $N_{x}, N_{y}$ and $N_{h}$ are the number of mesh elements in $x$ - and $y$-direction, and harmonics, respectively. Then, the magnetic field strength in $x$-direction is integrated over the interface for both MEC and FA expressions. Finally, the sine and cosine terms of each harmonic of both regions are equalized. The resultant equations are used in the model

$$
\begin{aligned}
& -\frac{2}{w_{n} \tau_{p}} \sum_{j=1}^{N}\left[( \frac { \phi _ { x - } ^ { i , j } + \phi _ { x + } ^ { i , j } } { 2 \mu _ { 0 } \mu _ { r } ^ { i , j } l ( y _ { + } ^ { i , j } - y _ { - } ^ { i , j } ) } ) \left(\cos \left(w_{n} x_{+}^{i, j}\right)\right.\right. \\
& \left.\left.-\cos \left(w_{n} x_{-}^{i, j}\right)\right)\right]=\frac{c_{n} e^{w_{n} y}-d_{n} e^{-w_{n} y}}{\mu_{r}^{\mathrm{FA}} \mu_{0}} \\
& \frac{2}{w_{n} \tau_{p}} \sum_{j=1}^{N}\left[( \frac { \phi _ { x - } ^ { i , j } + \phi _ { x + } ^ { i , j } } { 2 \mu _ { 0 } \mu _ { r } ^ { i , j } l ( y _ { + } ^ { i , j } - y _ { - } ^ { i , j } ) } ) \left(\sin \left(w_{n} x_{+}^{i, j}\right)\right.\right. \\
& \left.\left.-\sin \left(w_{n} x_{-}^{(i, j)}\right)\right)\right]=\frac{a_{n} e^{w_{n} y}-b_{n} e^{-w_{n} y}}{\mu_{r}^{\mathrm{FA}} \mu_{0}} .
\end{aligned}
$$

\section{B. Fixed-Point Method (FPM)}

In FPM, the relative permeability and remanent flux density of each mesh element are updated based on a specific strategy. This strategy uses the modulus of the magnetic flux density at the mesh node and returns two parameters which are graphically illustrated in [12]: the derivative of the magnetic field strength with respect to the magnetic flux density modulus as the incremental permeability, $\mu_{r}$, and the intercept as the remanent flux density, $B_{r}$, which is an additional MMF term

$$
\mathcal{F}_{x-}^{\mathrm{BH}}(i, j)=\mathcal{F}_{x+}^{\mathrm{BH}}(i, j)=\frac{y_{+}^{i, j}-y_{-}^{i, j}}{2 \mu_{r}^{i, j} \mu_{0}} \frac{B_{x}^{i, j} B_{r}^{i, j}}{\left|B^{i, j}\right|},
$$

$$
\mathcal{F}_{y-}^{\mathrm{BH}}(i, j)=\mathcal{F}_{y+}^{\mathrm{BH}}(i, j)=\frac{x_{+}^{i, j}-x_{-}^{i, j}}{2 \mu_{r}^{i, j} \mu_{0}} \frac{B_{y}^{i, j} B_{r}^{i, j}}{\left|B^{i, j}\right|} .
$$

The additional MMF sources given in (15) and (16) are used together with the current related MMF sources in the system of nonlinear equations which is solved iteratively. In each iteration, $B_{r}$ and $\mu_{r}$ matrices are updated according to the magnetic flux density distribution. To estimate the error during the convergence of the nonlinear solver, different global or local parameters are used such as magnetic scalar potential, magnetic flux density, attraction force or flux linkage.

\section{RESUlTS}

Firstly, the nonlinear benchmark problem is solved by FEM with 21600 dof and triangular-shaped second-order mesh elements using Comsol Multiphysics 5.4 software. In order to determine the number of elements in different regions of the benchmark, a mesh convergence study is carried for FEM. The maximum size of the mesh elements near corners is determined as $0.05 \mathrm{~mm}$ to avoid having large errors in these regions. Then, the developed HAM is used with FPM nonlinear solver for the same problem. In both methods, the attraction force is calculated using the Maxwell stress tensor. It is applied to a surface $(S)$ enclosing one of the parts, but the contribution to the integral of the Dirichlet and periodic boundaries is equal to zero. The middle of the airgap is chosen to minimize the numerical noise

$$
F_{y}=\frac{1}{2 \mu_{0}} \oint_{S}\left(B_{y}^{2}+B_{x}^{2}\right) d S .
$$

Combining (17), (5), (6) and the advantage of having an even symmetric benchmark, $F_{y}$ is derived as

$$
F_{y}=\frac{l \tau_{p}}{\mu_{0}} \sum_{n=1}^{N_{h}} c_{n} d_{n}
$$

The absolute error is defined as the normalized difference between the attraction force calculated using HAM $\left(F_{\text {HAM }}\right)$ and FEM $\left(F_{\mathrm{FEM}}\right)$. The variation of the absolute error with respect to $N_{x}$ and $N_{y}$, and $N_{h}$ is presented in Fig. 3. While $N_{y}$ and $N_{h}$ are 60 and 50 respectively in Fig. 3(a), $N_{x}$ and $N_{h}$ are 120 and 50 respectively in Fig. 3(b). Moreover, in Fig. 3(c), $N_{x}$ and $N_{y}$ are set to 120 and 60, respectively. In addition, the current density $(J)$ is selected as $20 \mathrm{~A} / \mathrm{mm}^{2}$ to reach the nonlinear region of the magnetization curve of the iron material. The purpose of this analysis is to achieve an accurate hybrid analytical model and show the convergence of the attraction force predicted by HAM under different mesh and harmonic refinements. A discrepancy of less than $1 \%$ is accepted as sufficient engineering accuracy for electrical machine applications. The figure shows that $0.18 \%$ absolute error in the attraction force is achieved with $N_{x}=120, N_{y}=60$, and $N_{h}=50$. This shows that FPM used in HAM is able to obtain similar accuracy as state-of-the-art high-order methods [16]. On the other hand, it is also observed that analyzing further than that point causes the system of equation to be illconditioned, which leads to numerical errors in the solution. However, since the selected model parameter values provide acceptable accuracy, further investigation is not covered in 


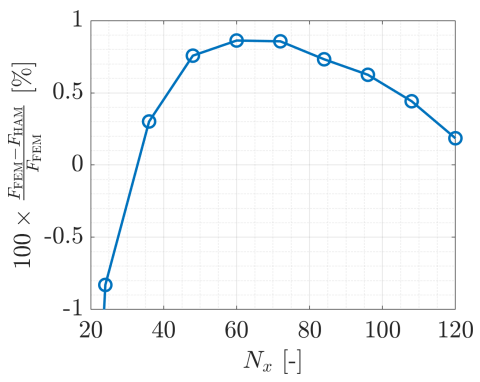

(a)

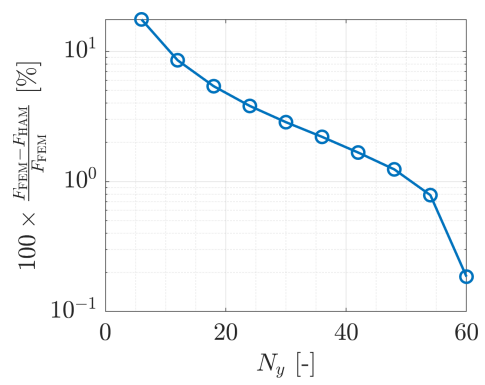

(b)

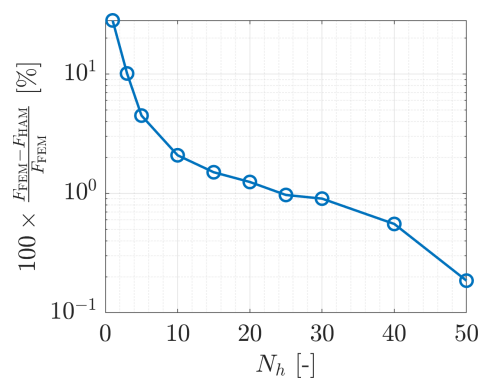

(c)

Fig. 3. Effect of the number of mesh elements in $x$ - and $y$-directions and harmonics on the absolute error of attraction force.

this study. After the refinements, HAM consists of 7200 MEC elements and 200 Fourier coefficients that corresponds to 7400 dof.

The investigation of local distributions is also essential to observe the absolute error for different sections of the benchmark. In Fig. 4(a), the root mean square (rms) of the error in the magnetic flux density distribution of the stator, mover and airgap is presented with respect to the number of dof. The rms of the magnetic flux density error is defined as

$$
\left(B_{\mathrm{HAM}}-B_{\mathrm{FEM}}\right)_{\mathrm{rms}}=\sqrt{\frac{1}{N} \sum_{k=1}^{N}\left(B_{\mathrm{FEM}}^{k}-B_{\mathrm{HAM}}^{k}\right)^{2}},
$$

where $N$ is the total number of points. For the stator and mover, the center of each mesh element is selected as a grid point. Since there is no mesh element in the airgap, the grid points are generated in the post-processing of HAM. In total, 600 grid points are used in the airgap in a way that the distance between the neighboring points is $0.1 \mathrm{~mm}$. Fig. 4(a) shows that the decrease of the magnetic flux density error of the stator is slower than others. The increase of the computational cost of a single iteration with respect to the number of dof is presented in Fig. 4(b). Moreover, it is observed that the computation time does not change with the change of current density.

In order to observe the convergence of the FPM, different error estimators are used in HAM. The relative difference between successive iterations for a global parameter such as force $\left(\varepsilon_{F}\right)$ and a local parameter such as the magnetic flux density $\left(\varepsilon_{B}\right)$ is expressed as

$$
\varepsilon_{F}^{(i)}=\frac{\left|F^{(i)}-F^{(i-1)}\right|}{F^{(i)}}, \varepsilon_{B}^{(i)}=\frac{\left\|B^{(i)}-B^{(i-1)}\right\|}{\left\|B^{(i)}\right\|} .
$$

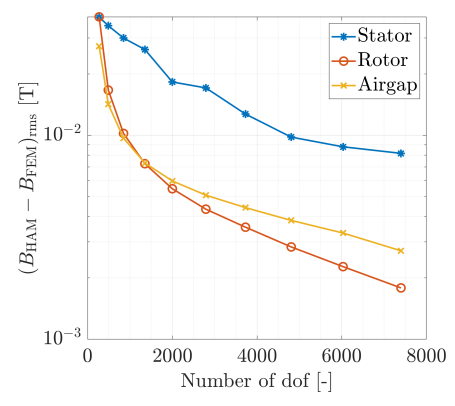

(a)

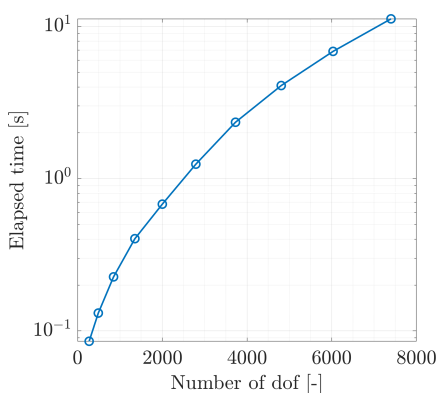

(b)
Fig. 4. The change of absolute error in magnetic flux density and elapsed time with the number of dof. where $\|\cdot\|$ is the L2-norm. The same approach of local parameters is applied to the magnetic scalar potential $(\psi)$ and Fourier coefficients (FC) for the same grid points used in Fig. 4(a) and the results are presented in Fig. 5(a) for 20 $\mathrm{A} / \mathrm{mm}^{2}$. In addition, the variation of the relative difference of the attraction force under different saturation levels is given in Fig. 5(b). In order to compare the speed of convergences, the rates are estimated by relative differences following

$$
\Delta F=\frac{\log _{10}\left(\varepsilon_{F}^{\left(i_{1}\right)}\right)-\log _{10}\left(\varepsilon_{F}^{\left(i_{2}\right)}\right)}{i_{2}-i_{1}},
$$

where $\Delta F$ is the convergence rate of the attraction force, $i_{1}$ and $i_{2}$ are the iteration indices. The initial (init) and average (ave) slopes of different error estimators for different current densities are calculated using (21) and presented in Table I. Fig. 5(a) shows that the convergence of the magnetic flux density is slower than the other error estimators. Fig. 5(b) and Table I show that the convergence rate decreases with the saturation. Table I shows that the convergence of any error estimator is faster at the beginning of the iterations compared to the rest. Fig. 5(b) shows that when the relative difference is smaller than $10^{-5}$, the numerical error due to the ill-condition number might disturb the smoothness of the convergence for some error estimators and excitation levels. However, the iterations can be stopped when the error estimators reach some prescribed threshold. For instance, $10^{-4}$ on the force estimator is sufficient for electrical machines applications. This corresponds to two fixed-point iterations in mildly saturated cases and four iterations for strongly saturated one.

Lastly, the resultant magnetic flux density distribution calculated using HAM for $20 \mathrm{~A} / \mathrm{mm}^{2}$ current density is presented in Fig. 6(a). The finite element approximation to the magnetic

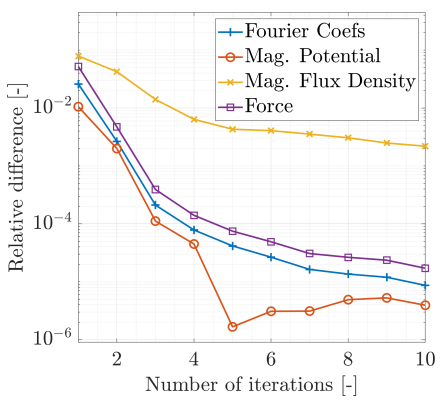

(a)

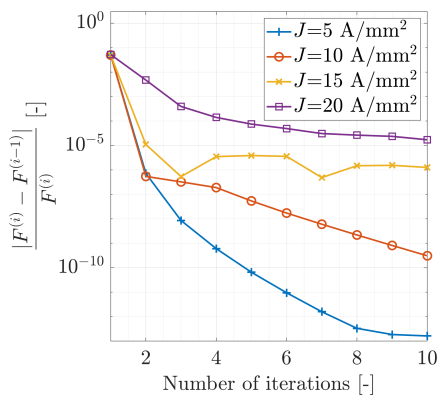

(b)
Fig. 5. Relative difference between successive iterations for different error estimators and excitation levels. 


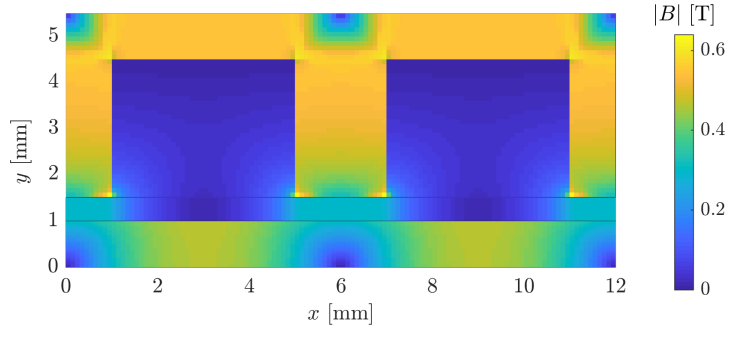

(a)

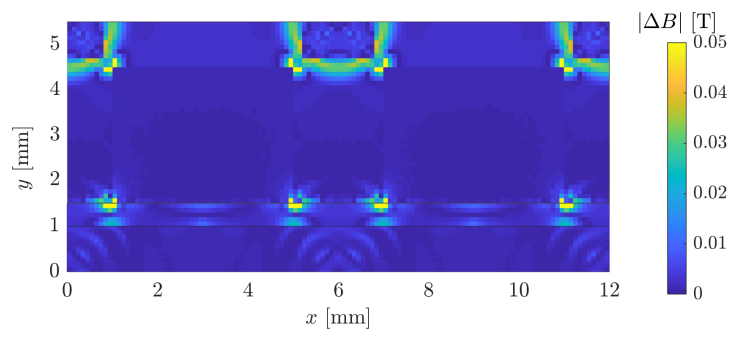

(b)

Fig. 6. Magnetic flux density distribution calculated using HAM and the difference between HAM and FEM.

TABLE I

INITIAL AND AVERAGE CONVERGENCE RATES WITH INCREASING CURRENT DENSITY FOR THE DIFFERENT ERRORS ESTIMATORS.

\begin{tabular}{|c|c|c|c|c|c|c|c|c|}
\hline$J$ & \multicolumn{2}{|c|}{$\Delta \psi$} & \multicolumn{2}{|c|}{$\Delta F$} & \multicolumn{2}{c|}{$\Delta F C$} & \multicolumn{2}{|c|}{$\Delta B$} \\
\cline { 2 - 9 }$\left[\mathrm{A} / \mathrm{mm}^{2}\right]$ & init & ave & init & ave & init & ave & init & ave \\
\hline 5 & 2.77 & 1.22 & 2.42 & 1.27 & 3.38 & 1.28 & 0.85 & 0.78 \\
10 & 2.03 & 0.89 & 2.48 & 0.91 & 2.58 & 0.91 & 0.46 & 0.36 \\
15 & 0.61 & 0.49 & 1.83 & 0.51 & 2.23 & 0.51 & 0.16 & 0.15 \\
20 & 0.36 & 0.38 & 0.52 & 0.39 & 1.06 & 0.39 & 0.17 & 0.14 \\
\hline
\end{tabular}

flux density is constructed by interpolation using the calculated solution vector of FEM. In Fig. 6(b), the magnetic flux density distribution of HAM is compared with the interpolated results of the FEM for the position of each MEC elements and the defined $0.1 \mathrm{~mm}$ grid in the airgap of HAM. The maximum error is obtained as $50 \mathrm{mT}$, while the maximum flux density in the benchmark is $0.6 \mathrm{~T}$. In addition, the rms discrepancy of the magnetic flux density is calculated as $9 \mathrm{mT}$ using (19). Since the global parameters, such as the attraction force or inductance, are the result of an integration, the effect of the maximum local error is negligible. Moreover, the maximum error in the flux distribution is located on the corners of the stator teeth. This is the main reason for the large rms error of the magnetic flux density in the stator (Fig. 4(a)) and the slow convergence of the magnetic flux density (Fig. 5(a)). To improve on this result, the adaptive mesh refinement method can be applied to the MEC regions to decrease the maximum local error on the corners by optimizing the mesh size.

\section{CONClusion}

The accuracy and convergence of HAM with FPM is analyzed for a 2-D magnetostatic problem. The proposed method is favorable to solve a wide spectrum of electromagnetic problems related to the reluctance machine including the magnetic saturation. It is shown that the Fourier coefficient, scalar potentials, and attraction force exhibit similar rates of convergence, while the convergence of the magnetic flux density is slower than the other proposed error estimators. Moreover, the initial convergence rate of the attraction force is decreased from 2.42 to 0.52 when the current density is increased from 5 to 20 $\mathrm{A} / \mathrm{mm}^{2}$. The reason is that a large electrical excitation results in high magnetic saturation which increases the saturation related MMF values in the reluctance network. FPM obtains the field solution by updating these MMF values iteratively. Hence, reaching the final distribution of the MMF is more time consuming under stronger magnetic saturation. Although the maximum discrepancy of the magnetic flux density is obtained as $50 \mathrm{mT}$ on the stator corners, the rms error including all domains of the benchmark is calculated as $9 \mathrm{mT}$. Lastly, it is demonstrated that an excellent agreement of the attraction force, $0.18 \%$, is obtained with the proposed approach with three times less degrees-of-freedom as compared to FEM.

\section{REFERENCES}

[1] W. N. Fu, P. Zhou, D. Lin, S. Stanton, and Z. J. Cendes, "Modeling of solid conductors in two-dimensional transient finite-element analysis and its application to electric machines," IEEE Trans. Magn., vol. 40, no. 2, pp. 426-434, Mar. 2004.

[2] S. Li and H. Hofmann, "Numerically efficient steady-state finite-element analysis of magnetically saturated electromechanical devices," IEEE Trans. Magn., vol. 39, no. 6, pp. 3481-3485, Nov. 2003.

[3] E. Dlala, A. Belahcen, and A. Arkkio, "A fast fixed-point method for solving magnetic field problems in media of hysteresis," IEEE Trans. Magn., vol. 44, no. 6, pp. 1214-1217, Jun. 2008.

[4] G. Koczka, S. Außerhofer, O. Bíró, and K. Preis, "Optimal convergence of the fixed-point method for nonlinear eddy current problems," IEEE Trans. Magn., vol. 45, no. 3, pp. 948-951, Mar. 2009.

[5] P. Zhou, D. Lin, C. Lu, M. Rosu, and D. Ionel, "An adaptive fixed-point iteration algorithm for finite-element analysis with magnetic hysteresis materials," IEEE Trans. Magn., vol. 53, no. 10, pp. 1-5, Oct. 2017.

[6] H. Gholizad, M. Mirsalim, and M. Mirzayee, "Dynamic analysis of highly saturated switched reluctance motors using coupled magnetic equivalent circuit and the analytical solution," in Proc. Int. Conf. Comput. Electromagn. (CEM), Aachen, Germany, pp. 1-2, Apr. 2006.

[7] E. Ilhan, B. L. J. Gysen, J. J. H. Paulides, and E. A. Lomonova, "Analytical hybrid model for flux switching permanent magnet machines," IEEE Trans. Magn., vol. 46, no. 6, pp. 1762-1765, June 2010.

[8] Y. Laoubi, M. Dhifli, G. Verez, Y. Amara, and G. Barakat, "Open circuit performance analysis of a PM linear machine using a new hybrid analytical model," IEEE Trans. Magn., vol. 51, no. 3, pp. 1-4, Mar. 2015.

[9] K. J. W. Pluk, J. W. Jansen, and E. A. Lomonova, "Hybrid analytical modeling: Fourier modeling combined with mesh-based magnetic equivalent circuits," IEEE Trans. Magn., vol. 51, no. 8, pp. 1-12, Aug. 2015.

[10] S. Ouagued, Y. Amara, and G. Bakarat, "Cogging force analysis of linear permanent magnet machines using a hybrid analytical model," IEEE Trans. Magn., vol. 52, no. 7, pp. 1-4, Jul. 2015.

[11] M. Desvaux, S. Sire, S. Hlioui, H. B. Ahmed, and B. Multon, "Development of a hybrid analytical model for a fast computation of magnetic losses and optimization of coaxial magnetic gears," IEEE Trans. Energy Convers., vol. 34, no. 1, pp. 25-35, Mar. 2019.

[12] J. Bao, B. L. J. Gysen, and E. A. Lomonova, "Hybrid analytical modeling of saturated linear and rotary electrical machines: integration of Fourier modeling and magnetic equivalent circuits," IEEE Trans. Magn., vol. 54, no. 11, pp. 1-5, Nov. 2018.

[13] W. Sun, Q. Li, L. Sun, L. Zhu, and L. Li, "Electromagnetic analysis on novel rotor-segmented axial-field SRM based on dynamic magnetic equivalent circuit," IEEE Trans. Magn., vol. 55, no. 6, pp. 1-5, Jun. 2019.

[14] S. Ouagued, Y. Amara, and G. Barakat, "Comparison of hybrid analytical modelling and reluctance network modelling for pre-design purposes," Math. Comput. Simul., vol. 130, pp. 3-21, Dec. 2016.

[15] B. L. J. Gysen, K. J. Meessen, J. J. H. Paulides, and E. A. Lomonova, "General formulation of the electromagnetic field distribution in machines and devices using Fourier analysis," IEEE Trans. Magn., vol. 46, no. 1, pp. 39-52, Jan. 2010.

[16] L. A. J. Friedrich, M. Curti, B. L. J. Gysen, and E. A. Lomonova, "High-order methods applied to nonlinear magnetostatic problems," Math. Comput. Appl., vol. 24, no. 19, pp. 1-15, Jan. 2019. 